\title{
Selección bibliográfica
}

\author{
Bibliographic Selection
}

\author{
Navarrete-Hernández $J .^{1}$
}

\section{Área Académica De Psicología}

Espósito DH. Influence of prevalent aesthetic body model and body dissatisfaction in high school students 2015;6:91-6.

Cortez D, Gallegos M, Jiménez T, Martínez P, Saravia S, Cruzat-mandich C, et al. Influence of sociocultural factors on body image from the perspective of adolescent girls. Rev Mex Trastor Aliment 2016;7:116-24. doi:10.1016/j.rmta.2016.05.001

Valdez-hernández V, Bojorquez I, Lourdes L De, Romo E, Unikel C. “ “You have to be slim !"' Epiphanies : Body image construction in middle-aged women. Rev Mex Trastor Aliment 2017;8:63-71. doi:10.1016/j.rmta.2017.01.003.

Andreea-elena MǴ. The risk of eating disorders in adolescence and its association with the value of the body mass index , level of anxiety and level of depression 2015;187:141-6. doi:10.1016/j.sbspro.2015.03.027.

Stice E, Marti CN, Durant S. Behaviour Research and Therapy Risk factors for onset of eating disorders : Evidence of multiple risk pathways from an 8year prospective study. Behav Res Ther 2011;49:622-7. doi:10.1016/j.brat.2011.06.009.

Berengüí R, Castejón MÁ, Soledad M. Body dissatisfaction , risk behaviors and eating disorders in university students. Rev Mex Trastor Aliment 2016;7:1-8. doi:10.1016/j.rmta.2016.02.004.

Betzaida M, Vizmanos B UC. Continuo de conductas alimentarias de riesgo en adolescentes de México. Rev Panam Salud Pública 2011;30:401-7.

Revisión N, Ojeda GA. Trastorno de la Conducta Alimentaria: Anorexia Nerviosa. Revisión bibliográfica. 2006;156:24-30.

Arévalo RV, Aguilar XL, Tellez-girón MTO, Mancilla-diaz JM, Los D. Eating disorders diagnostic : from the DSM-IV to DSM-5 2015:108-20.

[Acevedo SP, Saldaña RG, Hernández RL. A preliminary study of the predictive factors of binge eating behavior in three cultures: Mexico, Spain and Argentina. Rev Mex Trastor Aliment / Mex J Eat Disord 2013;4:68 78. doi:10.1016/S2007-1523(13)71994-3.

Valdés, M, Vila, A.. La parentalidad desde el parentesco: un concepto antropológico e interdisciplinar. Quadrens-e institut catalá d Antropologia, (2016); 21 (2): 4-20.

Calero, G., Quiñones, J., A., Martínez, P., M.. Adolescencia temprana y parentalidad. Fundamentos teóricos y metodológicos acerca de esta etapa y su manejo. Medisur. (2017);15(5): 1-7

Barudy, J., Dantagnan, M. Parentalidad, Buenos tratos y competencias parentales. En Barudy, J., Dantagnan, M. Los desafíos invisibles de ser madre o padre: manual de evaluacion de las competencias la resiliencia parental. 2013. Barcelona: 2010. pp 31-49. disponible en
https://books.google.com.mx/books?hl=es\&lr=\&id=PyUl BQAAQBAJ\&oi=fnd\&pg=PA15\&dq=Los+desaf\%C3\%A Dos+invisibles+de+ser+madre+o+padre:+manual+de+e valuaci\%C3\%B3n++de+las+competencias+y+la+resilie $\underline{\text { ncia }+ \text { parental\&ots }=H c T 6 c l 8 q g R \& \operatorname{sig}=Z 5 / v h Z N A c j h d q o z}$ 2w9cr6lur4F $4 \# v=$ onepage $\& q \& f=$ false

Hernández, L. Revisión teórica del concepto de competencias parentales. 2016. $1-22$

Martin, C.. La parentalidad: controversias en torno de un problema publico. Revista de estudios de Género. La ventana. 2005; (2): 7-30.

Torío, L. S., Peña C. J. V., Rodríguez M. M.. estilos educativos parentales. revisión bibliográfica y reformulación teórica. Teoría de la Educación. 2008; 20: 151-178.

Muñoz, S. A. La familia como contexto de desarrollo infantil. Dimensiones de análisis relevantes para la interacción educativa y social. Portularia. 2005 5 (2): $147-163$

Haces, M., A. Significado y ejercicio fe los roles parentales entre varones homosexuales. Revista de Estudios de Género. La ventana.2006; 23: 127 165 .

Bustos, B. Roles, Actitudes y Expectativas de Género en la Vida Familiar. La ventana. 1999;(9): 130-157

Di marco, G.. La transformación de los modelos de género y la democratización de las familias. En: Schmukler, B., Di Marco, G. Madres y democratización de la familia en la argentina contemporánea. Segunda. Argentina: Biblos; 1997. 37-46. Disponible en https://books.google.com.mx/books?id=085f6jxvlc8C\&p $g=P A 7 \&$ source $=g b s$ selected pages\&cad $=3 \# v=0$ nepa ge\&q\&f=false

Amarís, M., Camacho, R., Fernández, I. El rol del padre en las familias con madres que trabajan fuera del hogar. Psicología desde el caribe. 2000; 5: $157-175$

Valdés, E. Estilos parentales, participación de los padres en la escuela y ajuste académico de los alumnos de secundaria. [Tesis de Licenciatura]. México: Universidad Nacional Autónoma de México; 2015.

Hernández, C. M. Prácticas parentales y su relación con la adaptación en la adolescencia. [Tesis de Licenciatura]. México: Universidad Nacional Autónoma de México. 2015.

Kim, C., Yang, Z., Lee, H.. Parental style, parental practices, and socialization outcomes: An investigation of their linkages in the consumer socialization context. Journal of Economic Psychology .2015; 49: 15-33.

Rosas, T. L. Prácticas parentales asociadas al debut sexual prematuro. [Tesis

\footnotetext{
${ }^{1}$ Autor de Correspondencia. Instituto de Ciencias de la Salud, Universidad Autónoma del Estado de Hidalgo, Pachuca de Soto. México. Email:
} josedejesus_navarrete@uaeh.edu.mx 
de Licenciatura]. México: Universidad Nacional Autónoma de México. 2015.

Vicente, X. Influencia de las practicas parentales y ansiedad juvenil en la conducta autolesiva del adolescente. [Tesis de Doctorado]. México: Universidad Nacional Autónoma de México. 2015.

Andrade, P., \& Betancourt, D. Escala Prácticas Parentales para Adolescentes (PP-A). México DF, México: Universidad Nacional Autónoma de México; 2008.

Cortez, A. , González, M. F.. Practicas parentales y resiliencia en niños de 5 a 12 años en una escuela primaria publica de la ciudad de México. [Tesis de Licenciatura]. México: Universidad Nacional Autónoma de México. 2017.

Rodríguez, N. G.Practicas parentales y habilidades sociales en los niños. [Tesis de Licenciatura]. México: Universidad Nacional Autónoma de México. 2015.

González, C., Guevara., Jiménez D., Alcázar R. Influencia de prácticas parentales sobre el nivel de asertividad, agresividad y rendimiento académico en adolescentes. European Scientific Journal. 2017; 13 (20): $37-54$

Malander, N. M. Percepción de prácticas parentales y estrategias de aprendizaje en estudiantes secundarios. Revista de psicología (Santiago). 2016; 25(1): 01-19.

Andrade, P., Betancourt, D., Vallejo, A., Ochoa, C., Segura, B., Rojas, R. M. Prácticas parentales y sintomatología depresiva en adolescentes. Salud mental. 2012; 35(1): 29-36.

Caycho, T. P.. Relación con los padres y estrategias de afrontamiento en adolescentes de Lima. Propósitos y Representaciones.2016; 4(1): 11-59.

Solís, M., C., Vidal, M., A.. Estilos y estrategias de afrontamiento en adolescentes. Revista de psiquiatría y salud mental hermilio valdizan.2006; 7(1): 33-39.

Folkman, S.. Personal control and stress and coping processes: a theorical analysis. Journal of personality and social psychology. 1984; 46(4): 39852.

Burke, R. J. (s/f). Estilos de afrontamiento. En Sauter, S. L., Murphy, L. R., Hurrell, J. J. \& Levi, L. factores psicosociales y de organización.

Guevara, O. G, Hernández, V. H., Flores, L. T., Estilos de afrontamiento al estrés en pacientes drogadependientes. Revista de investigación en psicología. 2001; 4(1): 53-65.

Verdugo-Lucero, J. C., Ponce de león-Pagaza, B. G., Guardado-Llamas, R. E., Meda-Lara, R. M., Uribe- Alvarado, J. I., Guzmán-Muñiz, J.. Estilos de afrontamiento al estrés y bienestar subjetivo en adolescentes y jóvenes. Revista latinoamericana de ciencias sociales, niñez y juventud. 2013; 11(1): 79-91.

Richaud, D. M. C. Estilos parentales y estrategias de afrontamiento en niños. Revista latinoamericana de Psicología. 2005; 37(1): 47.

Rivera AS, Díaz LR, Méndez RF, Jaen CCI, García MM, Romero PA, Villanueva OGBT. 8 Escalas: El lado negativo de las Relaciones de Pareja. México: Manual Moderno; 2017.

\section{Área Académica De Enfermería}

Noguera O. (2011) "Embarazo en adolescente: Una mirada desde el cuidado de enfermería" Revista Colombiana de Enfermería. Vol 7 año 7. Pag 151-160

Martínez, Zoraida (1999). "Entre goces y amores", Documento inédito, Bogotá.

Romero S., Leonardo (2000)"La Educación sexual como un derecho", Pronunciamiento a favor de la Educación Sexual, Centro de Asesoría y Consultoría, Barranquilla - Colombia.

Castrillón, M. D. (2007). Discursos institucionales sobre la familia en Brasil y
Colombia: ¿biologizar/ nuclearizar, o reconocer su diversidad? Revista Latinoamericana de Ciencias Sociales, Niñez y Juventud, 5, 83-124.

Álvarez, M. 2011. Manual de Planeación Estratégica editor Panorama.

Goodstein, L. 2006.Planeación estratégica aplicada. Mc Graw Hill.

Híjar, G. 2011. Planeación Estratégica La visión prospectiva, Trillas

Lujambio, A. 2010. Modelo de Gestión Educativa Estratégica, 2a Ed. Programa Escuelas de Calidad, Alianza por la Calidad de la Educación. Gobierno Federal SEP. SNTE.

Manes, JM.2012. Gestión estratégica para Instituciones educativas. Gránica.

Munch. L. 2011. Planeación Estratégica planeación estratégica en el Modelo de Gestión Educativa, rumbo hacia el éxito. Trillas

\section{Área Académica De Medicina}

Hernández Medina M. S., Hernández Serrano C., López González A. L., Barragán López N., Islas Vega I., Cobián Díaz M. L., Reynoso Vázquez J., Ruvalcaba Ledezma J. C. American Journal of Public Health Research, 2017, Vol. 5, No. 5, 154-154. Available online at http://pubs.sciepub.com/ajphr/5/5/3

DOI:10.12691/ajphr-5-5-3.

Andrade, E. (2009). Ambientes de Aprendizaje para la Educación Tecnológica. [Electrónico] http://cvonline.uaeh.edu.mx/Cursos/Maestria/MTE/disenio_de_prog_de_ amb_de_apren/Unidad\%20II/amb_aprend_para_educ_tecnologica_Andr ade.pdf.

Pérez Escalante, G. (2014). Desarrollo de un ambiente de aprendizaje virtual como apoyo a la modalidad presencial en la asignatura de Taller de Informática de la Licenciatura en Administración de la Universidad Autónoma del Estado de Hidalgo. Maestría. Universidad Autónoma del Estado de Hidalgo.

Rodríguez Robledo, G. (n.d.). Ambientes de aprendizaje. [online] repositoriodigitalmaestriasunid. Available http://www.repositoriodigitalmaestriasunid.org/wpcontent/uploads/2013/11/Tema-1Ambientes-de-aprendizaje.pdf [Accessed 29 Mar. 2017].

World Health Organization. "Diabetes". [Noviembre 2017]. De: WHO. Sitio web: http://www.who.int/diabetes/en/

World Health Organization. "Global report on Diabetes". [Noviembre 2017]. De: WHO. Sitio web: http://www.who.int/diabetes/global-report/en/

Asociación latinoamericana de Diabetes. (2014). "Guía ALAD sobre el diagnóstico, control y tratamiento de la diabetes mellitus." México DF.

Borda M., Pérez G. (2000). "Manual de técnicas de modificación de conducta en medicina comportamental." España: Universidad de Sevilla.

Organización Mundial de la Salud. "Adherencia al tratamiento a largo plazo." [Noviembre 2017]. De: OMS. Sitio web: http://www1.paho.org/Spanish/AD/DPC/NC/adherencia-largo-plazo.pdf

Renders CM., Valk GD., Griffin SJ., Wagner E. (2000). "Interventions to improve the management of diabetes mellitus in primary care outpatient and community settings." Cochrane Database of Systematic Reviews, Issue 4. Art No:CD001481

Veliz M, Cárdenas A. (2005). "Calidad de Vida del paciente con Diabetes Mellitus Tipo 2”. México. (353)

Badía X. (2009). "Adherencia y cumplimiento terapéutico como mecanismo esencial para evitar el fracaso terapéutico de los pacientes". [Noviembre 2017]. Sitio web: http://www.correofarmaceutico.com/2009/11/30/al$\mathrm{dia} /$ entorno/la-adherencia-terapeuticarequiere-un-enfoque-integral-ymultidisciplinar

Bond WS, Hussar DA. Detection methods and strategies for improving medication compliance. Am J Hosp Pharm 1991; 48: 1978-1988. 
Organización Mundial de la Salud (Internet). Temas de Salud: Depresión. Online revisado el 2 de Marzo de 2018. Disponible en: http://www.who.int/topics/depression/es/

Flores Ocampo R.; Jiménez Escobar S, Pérez Hernández S., Ramírez Serrano P., Vega Valero C., Depresión y ansiedad en estudiantes universitarios. Revista electrónica de psicología Iztacala, 2207, Vol. 10 No.2, Pp. 94 104.

Instituto nacional de la salud mental, depresión. Consultado 02 de marzo, 2018. En línea en: https://www.nimh.nih.gov/health/publications/espanol/depresion/index.s html.

Bogert García H. La depresión etiología y tratamiento. Cienc Soc (Rep Dom) 2012; 17 (2): 183-197

Alberdi Sudupe J., Taboada O., Castro Dono C., Vázquez Ventosos C. Depresión. Médicos especialistas en Psiquiatría. Servicio hospitalario "Juan Canalejo", España. Guías clínicas 2006; 6.

Guerrero López J, Heinze Martin G, Ortiz de Leon S, Cortes Morelos J, Barragán Pérez V, Flores Ramos M. Factores que predicen la depresión en estudiantes de medicina. Gac med de Mex (Mex) 2013: 598-604.

González Olaya H, Delgado Rico H, Escobar Sánchez M, Cárdenas Angelone M. Asociación entre el estrés, el riesgo de depresión y el rendimiento académico en estudiantes de los primeros semestres de un programa colombiano de medicina. FEM (Col) 2014; 17(1): 47-53

Gutiérrez Rodas J, Montoya Vélez L, Toro Isaza B, Briñón Zapata M, Rosas Estrepo E, Salazar Quintero L. Depresión en estudiantes universitarios y su asociación con el estrés académico. CES Med 2010; 24(1): 7-17.

Osorno Castillo L, Palomino Garibay L. Depresión en estudiantes universitarios. Arch Med Fam 2009; 11 (1): 1-2.

Miranda Bastidas C, Gutiérrez Segura J, Bernal F, Escobar A., Prevalencia de depresión en estudiantes de medicina de la universidad del valle. Rev Colomb Psquiatr 2000; 29(3): 251-260

Arrivillaga Quintero M, Cortés García C, Goicochea Jiménez V, Lozano Ortiz T. Caracterización de la depresión en jóvenes universitarios. Univ Psychol 2004; 3(1): 17-26.

Smith CK, Peterson DF, Degenhardt BF, Johnson JC. Depression, anxiety, and perceived hassles among entering medical students. Psychol Health Med 2007; 12 (1):31-39.

Ferrel-Ortega R, Celis-Barros A, Hernández-Cantero O. Depresión y factores sociodemográficos asociados en estudiantes universitarios de ciencias de la salud de una universidad pública (Colombia). Psicol caribe 2011; 27: 40-60

Fernández C, Giraldo HD. Prevalencia del trastorno depresivo y factores asociados a esta patología en los estudiantes de pregrado del programa de Medicina de la Universidad de Caldas, en el segundo semestre del año 2000. Manizales: Universidad de Caldas, Facultad de Ciencias para la Salud, Departamento de Salud Mental y Comportamiento Humano; 2001.

Bohórquez Peñaranda A. Prevalencia de depresión y ansiedad según las escalas de Zung, y evaluación de la asociación con el desempeño académico en los estudiantes de medicina de la pontificia universidad Javeriana. (Tesis maestría). Bogotá DC: Facultad de Medicina, Maestría en Epidemiología Clínica, Pontificia Universidad Javeriana; 2007.

Cea Kremer K. Detección de depresión en estudiantes que ingresan a la facultad de medicina, universidad Austral de Chile, primer semestre año 2006 (Tesis pregrado). Valdivia: Universidad Austral de Chile, Facultad de Medicina, Escuela de Enfermería; 2006

López Roldán P y Fachelli S. Metodología de la investigación social cuantitativa. [Internet]. Barcelona, España. 2015. [Consultado el 20 de noviembre de 2017]. Disponible en: https://ddd.uab.cat/pub/caplli/2016/163567/metinvsoccua_a2016_cap23.pdf
Prieto G, Delgado AR. Fiabilidad y Validez. Papeles del Psicólogo. 2010;31(1):67-74

Morgan Lora P, Zárate Aguilar J, Ludeña Saldaña LH. Elaboración de instrumentos de evaluación de competencias profesionales. [Internet]. Lima, Perú. 2017. [Consultado el 20 de noviembre de 2017], Disponible en: https://www.sineace.gob.pe/wp-content/uploads/2017/03/03-Guiaelaboracion-Instrumentos-evaluacion.pdf

Escofet A, Folgueiras P, Luna E, Palou B. Elaboration and validation of questionnaire for the evaluation of service-learning projects. RMIE. 2016; 21(70):929-949.

Soriano Rodríguez AM. Diseño y validación de instrumentos de medición. Diálogos. 2014;14:19-40

Caravaca Morena JA, Noh S, Hamilton H, Brands B, et al. Factores socioculturales y consumo de drogas entre estudiantes universitarios costarricenses. 2015; 25(Esp):145-155.

Domenech D, Mann R, Strike C, Brands B, Khenti AU. Estudio de la prevalencia de la comorbilidad entre el distrés psicológico y el abuso de drogas en usuarios del Portal Amarillo, Montevideo -Uruguay. Texto Contexto Enferm [Internet]. 2012 [Consultado el 11 de noviembre de 2017]. Disponible en: http://www.scielo.br/scielo.php?pid=S010407072012000500022\&script=sci_arttext

Nunes ED. United Nations office on drugs and crime (UNODC). Global Study on homicide: trends,context, data. Vienna: UNODC; 2011. Ciênc Saúde Coletiva [Internet]. 2012 [Consultado el 11 de noviembre de 2017] Disponible en: http://www.scielo.br/pdf/csc/v17n12/29.pdf

Sánchez-Hernández, C., et al. Tabaquismo entre universitarios: caracterización del uno en la visión de los estudiantes. Rev. Latino-Am. Enfermagem. 2011; 19. 730-737

Organización Mundial de la Salud (OMS). Tabaco. [Internet]. 2017. [Consultado el 28 de Octubre de 2017] Disponible en: http://www.who.int/mediacentre/factsheets/fs339/es/

Cheesman, S., et al. Tabaquismo en estudiantes de medicina de la universidad de San Carlos de Guatemala. Revista Cubana de Salud Pública. 2015 41(1):18-32

Organización Mundial de la Salud (OMS). Alcohol. [Internet]. 2017. [Consultado el 28 de Octubre de 2017] Disponible en http://www.who.int/mediacentre/factsheets/fs349/es/

Organización Mundial de la Salud (OMS). Actividad física. [Internet]. 2017. [Consultado el 11 de noviembre de 2017]. Disponible en: http://www.who.int/dietphysicalactivity/pa/es/

Varela, M., et al. Actividad física y sedentarismo en jóvenes universitarios en Colombia: prácticas, motivos y recursos para realizarlos. Colomb Med. 2011; 42:269-277

Laing C, Chao K.M y Robinson, A. Managing the expectations of non-tradition students: a process of negotiation. Journal of Further and Higher Education. 2015; 29: 169-179.

Encuesta Nacional de Adicciones (ENA). 2011. Instituto Nacional de Salud Pública.

Mantilla S y Gómez-Conesa A. El cuestionario Internacional de Actividad Física. Rev Iberoam Fisioter Kinesol. 2007; 10 (1): 48-52

Alarco JJ, Álvarez-Andrade EV. Google Docs: una alternativa de encuestas online. Educ Med. 2012; 15(1):9-10

Ji Z, Gacía Seoane J, Vázquez T, et al. Empleo de Google Drive en evaluación universitaria. En: V encuentro sobre experiencias innovadoras en la docencia. Madrid, España. 2014. p 1-14

Xifra FJ, Losinno MA y Minetti ML. La encuesta digital, usos y herramientas. Escritos en la Facultad. 2013; 90:115-116

Díaz de Rada. Ventajas e inconvenientes de la encuesta por Internet. Papers. 2012; 97(1):193-223 
Caballeros-Camarena, A., et al. Tabaquismo en estudiantes de medicina: prevalencia y conocimientos de sus consecuencias. Rev Méd. 2013; 4 (3):171-173

Sánchez, M., et al. Consumo de tabaco en universitarios. Tesis de grado. Universidad Veracruzana. Minatitlán, Veracruz. 2007.

Santes-González, A., et al. Prevalencia y factores asociados al consumo de alcohol en estudiantes de medicina de primer y quinto semestre del Centro Universitario de Ciencias de la Salud de la Universidad de Guadalajara. Revista Médica. 2013; 5(1):22-26.

Amaro-Navarrete, C., et al. Factores sociodemográficos relacionados al patrón de consumo de alcohol en estudiantes universitarios del Área de Ciencias de la Salud de la Universidad de Nayarit. Revista Tamé. 2013;1(3):71-78.

Araiza, L., et al. Hábitos en el consumo de alcohol en el estudiante universitario y las consecuencias en su desarrollo académico. XVII congreso internacional sobre innovaciones en docencia e investigación en ciencias económico administrativas. Universidad Autónoma de Chihuahua, México. 2014.

Organización Mundial de la Salud (OMS) Dirección: http://www.who.int/dietphysicalactivity/factsheet_inactivity/es/ Actualización: 01/01/2015 Acceso: 30/04/2015

Sánchez-Contreras, M., et al. 2009. Factores de riesgo cardiovascular en poblaciones jóvenes. Rev Salud Pública. 11(1):110-122

Gil Madrona P, González Villora S, Pastor Vicedo JC y Fernández Bustos JG. Actividad física y hábitos relacionados con la salud en los jóvenes: estudio en España. Reflexiones. 2010; 89(2):147-161

García-Laguna DG, García-Salamanca GP, Tapiero-Paipa YT y Ramos C DM. Determinantes de os estilos de vida y su implicación en la salud de los jóvenes universitarios. Hacia la promoción de la salud. 2012; 17(2):169185.

Organización Mundial de la Salud. OMS: Las 10 principales causas de defunción. [Internet]. OMS. [Consultado el 6 de Octubre de 2017]. Disponible en: http://www.who.int/mediacentre/factsheets/fs310/es/

Hernández Ávila M, Rivera Dommarco J, Shamah Levy T, Cuevas Nasu L, Gómez Acosta LM, Gaona Pineda EB, et al. Encuesta Nacional de Salud y Nutrición 2016 de Medio Camino: Informe Final de Resultados. Primera. Hernández Ávila M, Rivera Dommarco J, Shamah Levy T, Cuevas Nasu L, Gómez Acosta LM, Gaona Pineda EB, et al., editors. Vol 2016. Cuernavaca, México: Instituto Nacional de Salud Pública; 2016.

Soto-Estrada, Moreno-Altamirano y Pahua-Díaz. Panorama epidemiológico de México, principales causas de morbilidad y mortalidad. Revista de la Facultad de Medicina de la UNAM. 2016; 59(6).

Gómez-Dantés O, Alonso-Concheiro A, Razo-García C, et al. Prioridades de investigación en Salud en México. Instituto Nacional de Salud Pública. Cuernavaca. 2017.

E. Eizayaga, D. Me. Homeos.org. Revisado 21 November 2017, Sitio web: http://homeos.org/wpcontent/uploads/2014/08/En que consiste la homeopatia.pdf

Déniz Octavio. Homeopatía . En Psico Homeopatía: Remedios para la mente y el corazón. 2007. (6-8). Sitio web: https://www.amazon.com.mx/Psico-HomeopatiaRemedios-Para-MenteCorazon/dp/1847535437/ref=sr 1 2?s=books\&ie=UTF 8\&qid=15194

Fernández Pérez Jorge A., Barajas Arroyo Guadalupe, Alarcón Pérez Lilia Mercedes. La enseñanza y la práctica de la homeopatía. Propiedad legítima y exclusiva del médico homeópata. de Revista de educación y desarrollo, 2008. 2 Sitio http://www.cucs.udg.mx/revistas/edu desarrollo/anterio res/9/009 Fernandez.pdf
Secretaría de Salud. Homeopatía en México. 04-09-2017, de Secretaría de Salud 2016. Sitio web: https://www.gob.mx/salud/acciones-yprogramas/homeopatia-en-mexico

Dra. Calleja, C. Perfil del paciente que consume homeopatía. Noviembre 20, 2017, de Ayuntamiento de Santander y Universidad de Cantabria, 2000. Sitio web: http://www.homeopatia.net/datos/pdf/perfil.pdf

Libro blanco de la homeopatía. Boiron.es. 2012. Retrieved 21 November 2017, from $\underline{h t t p: / / w w w . b o i r o n . e s / s i t e r e s o u r c e s / f i l e s / 5 / 94 . p d f ~}$

Eizayaga X., Treaty of Homeopathic Medicine. Argentina: Ed. Marecel, 1972

Ernesto Zedillo. Reglamento de insumo para la salud. Constitución Política de los Estados Unidos Mexicanos. 1998. Sitio web: http://www.salud.gob.mx/unidades/cdi/nom/compi/ris.ht $\underline{m l}$

Ruvalcaba Ledezma J.C, Peña Cisneros E.M, Segura Levi F, Monrroy Pelcastre J. La medicina de la persona "Bases conceptuales filosóficas" Pilares. 2012. 2(3): 16-17

Torres Barajas D, Flores morales T.C, Morales Cruz F, Ruvalcaba Ledezma J.C. Calidad de la atención en la consulta homeopática de la Zona Metropolitana de Guadalajara, México. 2005-2006. El Homepathic Heritage Internacional, 2010. 2 ( 2 y 3). 31-41

Aguilar, J. \& Mayén, B.. (1999). Métodos anticonceptivos para adolescentes. Salud sexual y reproductiva de los adolescentes, 3, pp. 25-32.

Tandazo, M. S. \& Catillo, M. B., . (2009). Descripción del conocimiento sobre métodos anticonceptivos y prevalencia de embarazo en las adolescentes de los colegios de la parroquia malacatos. Universidad técnica particular de loja escuela de medicina, 1, pp. 13-21.

Association of Reproductive Health Professional, Sexuality and Contraception, quick reference guide for clinicians. Updated July 2013

CONAPO secretaria de gobernación. 26 de Septiembre, Día Mundial para Prevención del Embarazo No Planificado en Adolescentes, 26 de septiembre de 2013

Villalobos, A., Allen, B., Hernández, M., Suárez, L., De la Vara, E. \& De Castro, F.. (2012, noviembre). Uso de anticonceptivos y planificación familiar entre mujeres adolescentes y adultas: cerrando la brecha entre metas y realidades. Instituto Nacional de Salud Pública, 5, pp. 41-56.

Indicadores seleccionados: Seguimiento de las metas de la CIPD Estado de La población mundial 2010. Indicadores seleccionados: Seguimiento de las metas de la CIPD Estado de La población mundial 2010

Moscoso, M., Rosario, R. \& Rodríguez, L., (2001). "Nuestra juventud adolescente: ¿cuál es el riesgo de contraer VIH?”. Revista Interamericana de Psicología, 35(2), pp. 79-81.

Mosquera, J., \& Mateus, J., (2003). Conocimientos, actitudes y prácticas sobre métodos de planificación familiar, VIH/SIDA y el uso de los medios de comunicación en jóvenes. Colombia médica, 34(4), pp. 206-212.

Fernández, R., López, L., Martínez, H., Kopecky, D., Uzcátegui, G. \& Muñoz, M.. (2009). Métodos anticonceptivos: prevalencia de uso según método en el Hospital "Materno Infantil del Este". Revista Obstetrica Ginecológica de Venezuela, 1, pp. 28-34.

Vázquez, A. L. \& Suárez, N.. (2006). Conocimiento y uso de métodos anticonceptivos en la población de 15 a 44 años del área urbana, Cunduacàn. Tabasco. Revista horizonte sanitario, 5 , pp.

Sondick, J. E., Madanns, J. \& Rothwell, J. C.. (2010). Use of Contraception in the United States. Vital and Healt Statistics, 23, pp. 1-54.

Maroto, A., Moreno, A., Rubio, M., Ortiz, C. \& Escobar, F.. (2012). Conocimiento y uso de métodos anticonceptivos por la población femenina de una zona de salud. Revista de Salud Pública, 1, pp. 21- 28.

Montero, A.. (2011). Anticoncepción en la adolescencia. Revista Médica Clínica, 1, pp. 59-67. 
Maiztegui, L. C.. (2006). Conocimientos Sobre Métodos Anticonceptivos Y Conductas De Salud Sexual Y Reproductiva De Las Mujeres Del Hospital Materno Provincial De Cordoba. Universidad Nacional de Cordoba Escuela De Salud Pública., 1, pp. 1-75.

Chen,J., Franklyn N., Judson, J. \& Douglas, M.. (1992). Barrier Contraceptives and Sexually Transmitted Diseases in Women: A Comparison of FemaleDependent Methods and Condoms. America Jural of public health, 82, pp. 669-664

Calzolari, E., Masciangelo, R., Milite, V. \& Verteram, R.. (200, febrero). Bacterial vaginosis and contraceptive methods. . International Journal of Gynecology \& Obstetrics., 1, pp. 341-346.

Cleto, M., Mota, C., Peguero, I., Peguero, M. \& Palacencio, M. . (2002). Conocimientos sobre métodos anticonceptivos en una población de estudiantes. Revista Médica Dominicana, 63, pp. 13-25.

Maturana, C. A., Álvarez, J. G., Carbonel \& Neira, J. (2009). Sexualidad y métodos anticonceptivos en estudiantes de educación secundaria. Revista Médica Peruana, 26, pp. 33-40.

Albeldaño,R. A. \& Sacchi,M.. (2011, diciembre). Comportamientos sexuales y uso de anticonceptivos en estudiantes universitarios. Revista de la Facultad de Ciencias de la Salud Universidad Nacional de Salta, 1, pp. 4 11.

L. Aragón, J. Ruiz, I. Cazali. (2011, enero.junio). Evaluación del conocimiento de VIH/SIDA en estudiantes preuniversitarios de la Ciudad de Guatemala. Revista de la Facultad de Medicina, 12, pp. 14-24.

Rachel Phelps, Patricia Murphy, Emily Godfrey. Choosing a Birth Control Michael J. Rosenberg MD, MPH, Arthur J. Davidson, MD, MSPH,

Cacéres, G. H., Echevarria, M. F., Gilarducci, C. G. \& Pomares, D. J. (2009, abril). Nivel de conocimientos sobre métodos anticonceptivos como futuros profesionales de la salud en estudiantes del último año de medicina. Revista de Posgrado de la VI a Cátedra de Medicina., 1, pp. 1523.

Jiménez, S., Andrade, P., \& Betancourt, D., (2007). Habilidades de comunicación en la conducta sexual de riesgo en jóvenes. Revista Intercontinental de Psicología y Educación, 9(002), pp. 147-162.

Cácera, G. H., Echeverria, M. F., Gilarducchi, C. \& Pomares, D. J.. (2009). Nivel de conocimientos sobre métodos anticonceptivos como futuros profesionales de la salud en estudiantes del último año de medicina. Revista de Posgrado de la Cátedra de Medicina, 8, pp. 22-31.

Rosenberg, M. J. \& Gollub, E. L. . (2011, marzo). Methods Women Can Use That May Prevent Sexually Transmitted Disease, Including HIV. American Jurnal of Public , 1, pp. 65-72.

María Guadalupe García Alcaraz; La educación sexual en la reforma educativa de los años setenta

Arteaga, M. S., Daza, S. M. \&Gómez, N., . (2006). Asociación entre conocimientos y uso de metodos anticonseptivos en estudiantes de enfermería y medicina de la universidad el bosque. Revista el Bosque, 1 , pp. 31-4228.- Gabriela Rodríguez. Educación sexual en el distrito federal

Gutiérrez Juan Pablo, López Zaragoza José Luis, Valencia Mendoza Atanacio, Pesqueira Eduardo, Ponce de León Samuel, Bertozzi Stefano M. (2004 Abr ). . Haciendo frente a la epidemia de VIH/SIDA en México: ¿Una respuesta organizada?. . Rev. invest. clín. [revista en la Internet], 56, 242252.

Secretaria De Educación pública: Educación Sexual en la Escuela y Género En la Educación; Programa de formación Curso-Taller Educación integral de la Sexualidad en la Escuela para Formadores de Docentes

Frenk, J., Ruelas, E., Tapia, R., León, M. E., González, E., Lomelín, G. \& Quintanilla, M. L. (2002). Introducción a los métodos anticonceptivos: Información general. Salud, Secretaría de Salud Dirección General de Salud Reproductiva, 3, pp. 10-15.

Mansour, D., Ink, P. \& Gemzel, K.. (2012, febrero). Sondick, J. E., Madanns,
J. \& Rothwell, J. C.. (2010, agosto). Use of Contraception in the United States. Vital and Healt Statistics, 23, pp. 1-54. . The European Journal of Contraception and Reproductive Health Care, 15, pp. 4-16.

\section{Área Académica De Odontología}

Buonocore MG. A simple method of increasing the adhesion of acrylic filling materials to enamel surfaces. JDENT RES. 1995 34:849-853.

Newman GV. Epoxy adhesives for orthodontic attachments: Progress report. Am J Orthod. 1965; 51:901-12.

Mitchell L. Decalcification during orthodontic treatment with fixed appliancesAn overview. Br J Orthod. 1992; 19:199-205.

Natera G, Alfredo E. Usos del rayo láser en Odontología restauradora. Primera parte. Aspectos generales, clasificación, interrelación con los tejidos vivos y precauciones en el uso. Acta Odontológica Venezolana. 2000; 38(1):110.

Sağır S, Usumez A, Ademci E, Usumez S. Effect of enamel laser irradiation at different pulse settings on shear bond strength of orthodontic brackets Angle Orthodontist. 2013; 83(6):973-80.

Pires PT, Ferreira JC, Oliveira SA, Azevedo AF, Dias WR, Melo PR. Shear bond strength and SEM morphology evaluation of different dental adhesives to enamel prepared with ER:YAG laser. Contemporary Clinical Dentistry. 2014; 4(1):20-6.

Martínez H. 2007. Odontología Láser, (eds.) Trillas: México, pp.11-91.

García González JE, Martínez Rodríguez FDC. El láser ¿motivación o realidad para el estudio de Física por los estudiantes de las Ciencias para la Salud? Revista Cubana de Investigación Biomédica. 2006; 25 (1): 1-6.

Oltra Arimon D, Berini Aytés AJ, Gay Escoda C. Aplicaciones del láser de baja potencia en odontología. RCOE. 2004; 9 (5):517-524.

Coluzzi DJ. Fundamentals of Lasers in Dentistry: Basic Science, tissue interaction, and Instrumentation. Journal of Laser Dentistry. 2008; 16 (Spec.Issue): 4-10.

Bader C, Krejcl I. Indications and limitations of Er: YAG laser applications in dentistry. American Journal of Dentistry. 2006; 19(3): 178-184

Cardoso MV, De Munck J, Coutinho E, Ermis RB, Van Landuyt K, de Carvalho RCR, Van Meerbeek B. Influence of Er,Cr:YSGG laser treatment on microtensile bond strength of adhesives to enamel. Operative Dentistry. 2008;33(4):448-55.).

Natera G, Alfredo E. Usos del rayo láser de Erbium: YAG (Er: YAG) EN Odontología restauradora. II Parte. Acta Odontológica Venezolana. 2002;40 (2):1-12.Orthod. 1965; 51:901-12. 29 Glenn VA. Erbium lasers in dentistry. Dent Clin N Am. 2004; 48:1017-1059.

Miserendino Let al. Lasers in Dentistry. Quintessence Books. Singapore, 1995; $161-4$.

Verma KS, Maheshwari S, Singh KR, Chaudhari KP. Laser in dentistry: An innovate tool in modern dental practice. Natl J Maxilofacial Surg. 2012; 3(2):124-132.

Graber TM, Vanarsdall RL, Vig KW. Ortodoncia. Principios y técnicas actuales. Cuarta edición. Editorial Elsevier Mosby. 2006; 579-620.

Sigüencia CV, García PA, Bravo CE. Estudio de la resistencia a la tracción de tres tipos de resinas fotopolimerizables para ortodoncia, en brackets metálicos a esmalte dental humano. Revista Latinoamericana de ortodoncia y Odontopediatría. 2014; 13(17) 6-14.

Seema K, Sharma-Sayal BS, Rossouw E, Gajanan V. The influence of orthodontic bracket base design on shear bond strength. Am JOrthod Dentofacial Orthop. 2003; 124 (1):74-82.

Jendresen MD, Glantz PO, Baier RE, Eick JD. Microtopography and clinical 
adhesiveness of an acid etched tooth Surface. Acta Odontol Scand. 1981; 39: $47-53$.

García BJ, Kessler NF. Adhesión en Patología y terapéutica Dental. Med. Síntesis. 1997; 87-96.

Gilpatrick RO, Ross JA, Simonsen RJ. Resin to enamel bond strengths with various etching times. Quintessence Int. 1991; 22: 47-52.

Camps A. La evolución de la adhesión a dentina. Avances en Odontoestomatología.2004; 20(1):11-17.

Daza Pulido LM, Sarmiento Bejarano LR, Güiza Cristancho EH. Determinación del patrón de grabado con láser y ácido ortofosfórico al 37\% sobre el esmalte dental. Univ Odontol. 2005; 25 (6): 31-40.

Contreras Bulnes R, Scougall Vilchis RJ, Rodríguez Vilchis LE, Centeno Pedraza C, Olea Mejía OF, Alcántara Galena MCZ. Evaluation of SelfEtching Adhesive and Er: YAG Laser Conditioning on the Shear Bond Strength of Orthodontic Brackets.The Scientific World Journal. 2013; (2013):1-5.

Pashley DH, Carvalho RM, Tay FR, Agee KA, Lee KW. Solvation of dried dentin matrix by wáter and other polars solvents. Am J DENT. 2002; 15(2): 97-102.

Ichnokawa T. Analytical scanning electron microscopy for solid Surface. J Electron Microsc Tech. 1989; 12 (3):219-27.

Izardy MI, Sherriff M, Cobourne MT. A comparative investigation into relative bond strengths of Damon3, Damon3MX, and APC II brackets using different primer and adhesive combinations. European Journal of Orthodontics. 2011; 10: 1-5.

Pithon MM, Dos Santos LR, Ruellas OC, Sant FE. One-component self-etching primer: a seventh generation of orthodontic bonding system?. European Journal of Orthodontics. 2010; 32(10): 567-570.

Caballero PA, Blincos UC, Fernández IJ, Rivera JR, Tanaka LE. Comparación de la fuerza de adhesión y el tipo de falla entre dos cementos de resina para ortodoncia. Univ. Odontol. 2011; 30 (65):31-39.

De Souza GA, Bergmann C, Prietsch JR, Vicenzi. Shear bond strength of metallic and ceramic brackets using color change adhesives. Dental Press JOrthod. 2013; 18(2): 76-80.

Hosseini MH, Namvar F, Chalipa J, Saber K, Chiniforush N, Sarmadi, Mirhashemi AH. Comparison of Shear Bond Strength of Orthodontic Brackets Bonded to Enamel Prepared By Er: YAG Laser and Conventional Acid-Etching. Journal od Dentistry.2012; 9(1):1-7.

Lerardo G, Di Carlo G, Petrillo, Luzzi V,Vozza I, Migliau G, Kornblit R, Rocca JP, Polimeni A. Er: YAG laser for brackets bonding: a SEM study after debonding. The Scientific World Journal. 2014;93(59):1-5.

Wright BW. Craniodental biomechanics and dietary toughnessin the genus Cebus. Journal of Human Evolution 2005;48:473-92.

Hallak SC, Santos CM, Vitti M, Regalo CA, Batista de Vasconcelos P, Wilson Mestriner WJ, et al. Evaluation of molar and incisor bite force in indigenous compared with white population in Brazil. Archives of Oral Biology. 2008;53(www.intl.elsevierhealth.com/journals/arob):282-86.

García EL. Diseño y Construcción de Electromiógrafo para el Registro de EMG Superficial de Músculos maseteros e Implementación del Análisis Mutifractal por DFA. Mexico D.F.: Centro de Investigación y de Estudios Avanzados del Instituto Politécnico Nacional; 2011.

Coelho-Ferraz MJ, Bërzin F, Amorim C, De Paula DG. Electromyographic Evaluation of Mandibular Biomechanic. Int J Morphol. 2009;27(2):48590.

Tecco S, Caputi S, Tete S, Orsini G, Festa F. Electromyographic activity of masticatory, neck and trunk muscles of subjects with different mandibular divergence. A cross-sectional evaluation. Angle Orthod. 2007 Mar;77(2):260-5.

Okenson J, editor. Oclusión y afecciones temporomandibulares. Madrid:
Harcourt-brace; 1999.

Harper RP, de Bruin H, Burcea I. Muscle activity during mandibular movements in normal and mandibular retrognating subjects. Oral Maxillofac Surg. 1997;3:225-33.

Deguchi I, Garetto LP, Sato Y, Potter RH, Roberts WE. Statistical analysis of differential lissajous EMG from normal occlusion and Class III malocclusion. Angle Orthod. 1955;55(3):151-60.

Duque S, Jaramillo F, Farbiarz P, Alvarez J, Peláez V, Otalvaro C, et al. Electromyographic changes after orthognathic surgery: report of four cases. Rev Fac Odontol Univ Antioquia. 2002;13(2):35-50.

Scherder E, Posthuma W, Bakker T, Vuijk PJ, Lobbezoo F. Functional status of masticatory system, executive function and episodic memory in older persons. Jorunal of Oral Rehabilitation. 2008;35:324-36.

Nicoline CW, Van der K, Maillow P, Jacobs J, Van der W, Machiel N, et al. Reproducible effects of subjectively assessed muscle fatigue on an inhibitory jaw reflex in humans. Archives of Oral Biology 2009;54:87983.

Brinkwhorth R, Turker KS, Sabundra AW. Response of human jaw muscle to axial stimulation of the incisor. Journal of Physiology. 2003;547:233-45.

Sowman PF, Turker KS. Periodontal masseteric reflexes decrease whit tooth pre-load. Journal Dental Research. 2008;87:175-9.

Sallen NM, Fueki K, Garret NR, Ohyama T. Objetive and subjetive hardness of a test item used for evaluating food mixing ability. Journal of Oral Rehabilitation 2007;34:227-34.

Bakke M, Handsdottir R. Mandibular function in patiens whit temporomandibular joint pain: a 3-year follow-up. Oral Surg 2008;106:227-34.

Lujan-Climent M, G.J. M, Palau S, Ayuso-Montero R, Salsench J, Peraire M. Influence of static and dynamic oclusal Characteristics and muscle force on masticatory performance in dentate adults. European Journal of Oral Sciences. 2008;116:229-36.

Hatch JP, Shinkai RS, Sakai S, Rugh JD, Paunovich ED. Determinats of masticatory performans in dentate adults Archives of Oral Biology. 2001;46:641-8.

Cecilio S, Hallak R. Evaluation of molar and incisor bite in indigenous compared whith white population in Brazil. Oral Biology. 2008;53:282-6.

Ikebe K, Nokubi T, Morji K, Jumpei K, Furuya M. Association of bite force whit ageing and occlusal support in older adults. Journal of Dentistry. 2005;30:131-7.

Okiyama S, Ikebe K, Nokubi T. Association between masticatory performance and maximal occlusal force in young men. Jorunal of Oral Rehabilitation. 2003;30:278-82.

Watanabe K. The relationship between dentofacial morphology and the isometric jaw-opening and closing muscle function as evaluated by electromyography. Journal Oral Rehabilitation. 2000;27:639-45.

Suvinen TI, Kemppainen P. Review of clinical EMG studies related to muscle and oclusal factors in healthy and TMD subjects. Jorunal of Oral Rehabilitation. 2007;34:631-44.

Proschel PA, Raum J. Preconditions for estimation of masticatory forces from dynamic EMG and isometric bite force activity relations of elevator muscles. Journal of Prosthodontics. 2001;14:563-9.

Clancy EA, Morin EL, Merletti R. Sampling, noise-reduction and amplitude estimation issues in surface electromyography. Journal of Electromyography and Kinesiology. 2002;12:1-16.

Farinaa D, Holobarb A, Merlettib R, Enokad RM. Decoding the neural drive to muscles frome the surface electromyogram. Clin Neurophysiol. 2010;121(10):1616-23.

De Luca CJ, Adam A, Wortiz R, Gilmore LD, Nawab SH. Descomposition of 
surface EMG signals. J Neurofhysiol. 2006;96:1646-56.

Caballero K, Duque L, Ceballos S, Ramirez J, Pelaez A. Conceptos básicos para el análisis electromiográfico. Revista CES Odontología. 2002;15(1):4150 .

Merletti R, Parker P. Electromyography: physiology, engineering and noninvasive applications. 1 ed: John Wiley \& Sons; 2004.

Alfaro P, Osorno C, Romero G. Evaluación neuromuscular masticatoria. Revista de Ciencias Clinicas. 2003;4(1):41-51.

Al-Mulla M, Sepulveda F, Colley M. A Review of Non-Invasive Techniques to Detect and Predict Localised Muscle Fatigue. Sensors [serial on the Internet]. 2011; (1424-8220).

Al-Mulla MR, Sepulveda F. Novel feature modelling the prediction and detection of sEMG

muscle fatigue towards an automated wearable system. Sensors. 2010;10:483854.

Gang W, Xiao-Mei R, Lei L, Zhi-zhong W. Multifractal analysis of surface EMG signals forassessing muscle fatigue during static contractions. J Zhejiang Univ-Sci A. 2007;8:910-15.

Hagberg M. Work load and fatigue in repetitive arm elevations. Ergonomics. 1981;24:543-55.

Petrofsky JS, Glaser RM, Phillips CA, Lind AR, Williams C. Evaluation of amplitude and frequency components of the surface EMG as an index of muscle fatigue. Ergonomics. 1982:213-23.

Kijak E, Lietz-Kijak D, Zbigniew C, Frączak B. Muscle activity in the course of rehabilitation of masticatory motor system functional disorders. Postepy Hig Med Dosw (online). 2013;67(1732-2693):507-16.

Bartuzi P, Danuta R. The Influence of Fatigue on Muscle Temperature. International Journal of Occupational Safety and Ergonomics 2012;18:233-43.

Ardizone I, Celemin A, Aneiros F, del Rio J, Sanchez T, Moreno I. Electromyographic study of activity of the masseter and anterior temporalis muscles in patients with temporomandibular joint (TMJ) dysfunction: comparison with the clinical dysfunction index. Med Oral Patol. 2010 Jan;15(1):e14-9.

Castroflorio T, Icardi K, Torsello F, Deregibus A, Debernardi C, Bracco P. Reproducibility of surface EMG in the human masseter and anterior temporalis muscle areas. Cranio. 2005 Apr;23(2):130-7.

Coelho-Ferraz M. Evaluación electromiográfica de los músculos maticadores durante la fuerza máxima de mordedura Rev Esp Cirug Oral y Maxilofac. 2008;30:420-27.

Naeije M, Lobbezoo F, Van der Kaaij N, Maillou P, Van der Weijden J. Reproducible effects of subjectively assessed muscle fatigue on an inhibitory jaw reflex in humans. Archives of Oral Biology. 2009;54:87983 .

Ferrario V, Sforza A, Miani A, D'Addona A. Electromyographic activity of human masticatory muscles in normal young people. Statistical evaluation of reference values for clinical applications. J Oral Rehabil. 1993;20:27180 .

Albornoz M, Ogalde A, Aguirre M. Estudio Radiográfico y Electromiográfico de los Músculos Masetero y Temporal Anterior en Individuos con Maloclusión Tipo II, 1 de Angle y Controles. Int J Morphol. 2009;27(3):861-66.

Robertson G, Caldwell B, Hamill J, Kamen G. Research methods in biomechanics. J Human Kinetics. 2004;3:163-80.

Ferrario VF, Tartaglia GM, Galleta A, Grassi GP. The influence of occlusion on jaw and neck muscle activity: a surface EMG study in healthy youn adults. Journal of Oral Rehabilitation. 2006;33:341-8.

Pisani M, de Sousa R, da Silva M, da Silva C. Electromyography of masticatory muscles after denture relining with soft and hard denture liners. Journal of Oral Science. 2013;55:217-24.

Davison L, Chiaron F, Sernik R, Tanaka C, Furquim C. Electromyographic and ultrasonographic assessment of the masseter muscle in normal individuals: a pilot study Pró-Fono Revista de Atualização Científica 2009;21:261-4.

De Felício CM, Pimenta FC, Magalhaes MA, Rodrigues DM, Tartaglia GM, Sforza C. Electomyographic indices, orofacial myofunctional status and temporomandibular disorders severity: A correlation study. Journal of Electromyography and Kinesiology. 2012;22:266-72.

Lowery M, O'Malley M. Analysis and simulation of changes in EMG amplitud during high-level fatiguing contractions. Trans Biomed Eng. 2003;50:1052-62.

Al-Mulla MR, Sepulveda F, Colley M, Kattan A. Classification of localized muscle fatigue with genetic programming on sEMG during isometric contraction. In: In Proceedings of Annual International Conference of the IEEE Eng. in Medicine and Biology Society EMBC. Minneapolis, MN U, editors. Minneapolis, MN, USA, 2009. p. 2633-8.

Talebinejad M, Chan AC, Miri A. Fatigue estimation using a novel multi-fractal detrended fluctuation analysys-based aproach. Journal of Electromyography and Kinesiology. 2010;20:433-9.

Rilo B, Santana U, Mora MJ, Cardaso CM. Myoelectrical activity of clinical rest position and jaw muscle activity in young adults. Journal of Oral Dentofacial Orthopedic. 1997;118:616-24.

Ueda HM, Miyamoto K, Saifuddin M. Masticatory muscle activity in children and adults with different facial types. J Orthod Dentofacial Orthop. 2000;118:63-8.

Berretin G, Nary H, Padovani C, Trindade A, Monteiro W. Electromyografic evaluation of mastication and swallowing in elderly individuals whit mandibular fixed implansupported prostheses. J Appl Oral Sci. 2008;16(2):116-21.

Fernández L, Zanotta G, Kreiner M. Estudio comparativo del complejo electromiográfico post-estímulo del músculo masetero en pacientes rehabilitados con prótesis completa bimaxilar mediante técnica piezográfica y técnica convencional. Odontoestomatologia. 2010;12(14):45-53.

Santos P, Trindade A, Sampaio A. Evaluación comparativa de la función masticatoria en individuos desdentados rehabilitados con prótesis soportadas por implantes óseo integrados. Rev Odontol Dominic. 2004;5(10):27-35.

Obrez A, Türp J. El efecto del dolor facial músculo-esqueletal en los registros de las relaciones maxilomandibulares y la planificación del tratamiento: Una síntesis de la literatura. The journalof prosthetic dentistry 2006;47(3):439-45.

Tetsuka M, Saga T, Nakamura M, Tabira Y, Kusukawa J, Yamaki K. Relationship between Masseter Muscle Form and Occlusal Supports of Remaining Teeth. Kurume Med J. 2012;59(1-2):5-15.

Angeles F, Navarro B, editors. Diseño en Prótesis Bucal Removible, procedimientos clínicos. México, D.F: Trillas; 1998.

Prosthodontics Ao. The glosary of Prosthodontic Terms. J Prosthet dent. $1994 ; 94$

Shifman A, Ben-Ur Z. The mandibular firstpremolar as an abutment for distal extension removable partial dentures: a modified clasp assembly desing. British Dent J.188:246-8.

Thompson JR. The rest position of the mandible and its significance to dental science. JADA. 1946;33:151-80.

Kapur KK. Studies of biologic parameters for denture design. Comparison of masseter muscle activity during chewing of crisp and soggy wafers in denture and dentition groups. J Prosthet dent. 1975;33:242.

Ingervall B, Hedegird B. An electromyographic study of masticatory and lip muscle function in patients with complete dentures. The Journal of 
prosthetic dentistry. 1980;43:266-71.

Tallgren A, Holden S, Lang BR, Ash MM. Jaw muscle activity in complete denture wearers. A longitudinal electromyographic study. Journal of Prosthetic Dentistry 1980:44-123.

Ikebe K, Nokubi T, Morii K, Kashiwagi J, Furuya M. Association of bite force with ageing and occlusal support in older adults. Journal of Dentistry. 2004;33:131-37.

Ingervall B, Hedegard B. An electromyographic study of masticatory and lip muscle function in patients with complete dentures. Journal of prosthetic dentistry 1980;43:266-71.

Ángeles MF, Nuño LA, González GH, García AA, García RJ. Refleximetría de los músculos maseteros: un método objetivo para la evaluación de la función masticatoria. Arch neurocien (Mex). 1997;2:215-23.

Castroflorio T, Icardi K, Becchino B, Merlo E, Debernardi C, Bracco P, et al. Reproducibility of surface EMG variables in isometric sub-maximal contractions of jaw elevator muscles. Journal of Electromyography and Kinesiology. 2006;16:498-505.

Hatch JP, Shinkai RSA, Sakai S, Rugh JD, Paunovich ED. Determinants of masticatory performance in dentate adults. Archives of Oral Biology. 2001;46:641-48.

\section{Área Académica De Nutrición}

Fuertes M. 2014. Extracción y cuantificación de inulina a partir del ajo. Tesis de Grado. Universidad Central del Ecuador. Facultad de Ingeniería Química. Quito.

García L. 2000. Revisión: Efectos cardiovasculares del ajo (Allium Sativum). Arch Latinoam Nutr. 50: 39-51.

Muñoz S, Restrepo D, Sepúlveda J. 2012. Revisión: Inulina en Algunos Derivados Cárnicos. Rev Fac Nal Agr Medellín. 65(2): 6789-6798.

Olagnero G, Abad A, Bendersky S, Genevois C, Granzella L, Montonati M. 2007. Alimentos funcionales: fibra, prebióticos, probióticos y simbióticos. Diate. 25(121): 20-33.

Roberfroid M. 2002. Functional Foods: concepts and application to inulin and oligofructose. Br J Nutr. 87: 139-143.

Roberfroid MB. 2005. Introducing inulin-type fructans. The British Journal of Nutrition. 93 (1): 13-25.

Rubio M. 2002. Implicaciones de la fibra en distintas patologías. Nutr Hosp. 17(2): 17-29.

Sánchez R, Martín M, Palma S, López B, Bermejo L, Gómez C. 2015. Indicaciones de diferentes tipos de fibra en distintas patologías. Nutr Hosp. 31(6): 2372-2383.

ATSDR. Resúmenes de Salud Pública - Hidrocarburos aromáticos policíclicos (HAP) [Polycyclic Aromatic Hydrocarbons (PHA)]. Agencia para Sustancias Tóxicas y el Registro de Enfermedades. Dirección: https://www.atsdr.cdc.gov/es/phs/es_phs69.html. Acceso: 20/10/2017.

Cross, A.J., \& Sinha, R. (2004). Mutagénicos y carcinógenos relacionados con la carne en la etiología del cáncer colorrectal. Environmental and Molecular Mutagenesis. 44(1):44-55.

Cruzar, J., Kish, V., \& Andriole, G. (2004). Estudio prospectivo de mutagénicos de carne y riesgo de cáncer de próstata. Cancer Research 2005. 65(24):11779-11780.

González, R. (2012). ¿POR QUÉ NO COMER CARNE? UNA MIRADA CIENTÍFICA

.Dirección: http://www.medicosconscientes.net/pdf/porque-no-comer-carne.pdf. Acceso: 20/10/2017.

Jägerstad, M., \& Skog, K. (2005). Genotoxicidad de los alimentos procesados térmicamente. Mutation Research. 574(1-2):156-157.
Katz, M. (2013).De las redes sociales al plato. En: ¿Somos lo que comemos? verdades y mentiras de la alimentación. 1a edición. AGUILAR. pp: 1-289.

Melgarejo, M.A. (2015). Consumo de carne y cáncer, controversias. Boletín Caipadi, 1-2.

Mendoza, E. (2012). Preparación de alimentos y formación de compuestos tóxicos. En: Toxicología de los alimentos. 1a edición. pp: 178-189.

Monckeberg, F. (2015). La reducción del consumo de carnes procesadas puede disminuir el riesgo de cáncer colorrectal y enfermedades crónicas Universidad de Chile. Dirección: https://inta.cl/es/opinion/la-reducciondel-consumo-de-carnes-procesadas-puede-disminuir-el-riesgo-de-cancer. Acceso: 25/09/2017.

OMS. Carcinogenicidad del consumo de carne roja y de la carne procesada. Organización Mundial de la Salud. Dirección: http://www.who.int/features/qa/cancer-red-meat/es/. Acceso: 20/10/2017.

Sugimura, T., Wakabayashi, K., Nakagama, H., \& Nagao M .(2004). Aminas heterocíclicas: Mutágenos/ carcinógenos producidos durante la cocción de carne y pescado. Cancer Science; 95(4):290.

Vulcan, A., Manjer, J., Ericson, U., \& Ohlsson. (2017). Intake of different types of red meat, poultry, and fish and incident colorectal cancer in women and men: results from the Malmö Diet and Cancer Study. Food Nutr Res. 61(1): 1-12.

Acevedo I, García O, Contreras J. y Acevedo I. Elaboración y evaluación de las características sensoriales de un yogurt de leche caprina con jalea semifluida de piña. Revista UDO Agrícola. 2009.12: 442-448.

Briones E.C. Elaboración de yogur. Universidad Autónoma del Estado de Hidalgo.2005. 1: 1-49.

Parra V.P. Estudio comparativo en el uso de colorantes naturales y sintéticos en alimentos desde el punto de vista funcional y toxicológico. Universidad Austral de Chile. 2004.1: 1-85.

Ibáñez F.C. Aditivos alimentarios. Universidad Pública de Navarra. 2003.1: 110

Centurión A, Solís S, Saucedo C, Báez R y Sauri E. Cambios físicos, químicos y sensoriales en frutos de pitahaya (hylocereus undatus) durante su desarrollo. Rev. Fitotec. Mex.2008. 31: 1-5.

Zhuang Y, Zhang y Sun L. Characteristics of fibre-rich powder and antioxidant activity of pitaya (Hylocereus undatus) peels. International Journal of Food Science \& Technology. 2012. 47: 1279-1285.

García-Cruz, Salinas-Moreno Y, Valle-Guadarrama S. Betalaínas, compuestos fenólicos y actividad antioxidante en pitaya de mayo (Stenocereus griseus H.). Revista fitotecnia mexicana. 2012.55: 1-5

SAGARPA.Pitahaya, la fruta del fuego y del dragón. [Internet] 2017 [actualizado 3 de Junio de 2017; citado 9 de oct de 2017] Disponible en: https://www.gob.mx/sagarpa/articulos/pitahaya-la-fruta-del-fuego-y-deldragon?idiom $=$ es

Moreu, B. La pitahaya. Farmacéutica y Tecnóloga de los Alimentos. Diplomada en Nutrición. [Internet] 2017 [actualizado 2017; citado7de oct de 2017] Disponible http://www.pulevasalud.com/ps/contenido.jsp?ID=60624\&TIPO_CONT ENIDO=Articulo\&ID CATEGORIA=90\&ABRIR SECCION=2\&RUT $\underline{\mathrm{A}=1-2-45-328}$

Ruvalcaba Ledezma, J.C., et al, (2017), Nutrición Básica-Clínica “Aprehender para una vida saludable", México, D.F.: Editorial Castdel, S.A de C.V. 